\title{
Non-tuberculous mycobacteria isolated from patients with suspected tuberculosis in Abidjan, Ivory Coast
}

\author{
*1,2Ouassa, T., ${ }^{1} \mathrm{~N}^{\prime}$ Guessan-Kacou, M. S., and ${ }^{2}$ Kouakou, K. A. \\ ${ }^{1}$ Centre for Diagnosis and Research on AIDS and other Infectious Diseases (CeDReS), \\ University Hospital of Treichville, Abidjan \\ ${ }^{2}$ Department of Bacteriology-Virology, Faculty of Pharmacy, University Félix Houphouët-Boigny, Abidjan \\ *Correspondence to: timothee.ouassa@cedres.org; timouassa@yahoo.fr; 0022521258459; 0022502500078
}

\begin{abstract}
:
Background: Apart from tuberculosis caused by Mycobacterium tuberculosis complex (MTBC) species, there are many other mycobacterial infections due to nontuberculous mycobacteria (NTM). These are rarely identified in many low resource settings in Africa because of the lack of accurate identification methods. The aim of the study is to identify NTM species involved in respiratory infections in Abidjan, Ivory Coast.

Methodology: Isolates routinely identified as NTM by the detection of MPT64 antigen between 2015 and 2018 at the Centre for Diagnosis and Research on AIDS and other Infectious Diseases (CeDReS) of the University Hospital of Treichville, were included in the study. Bacterial strains were sub-cultured on three different Lowenstein-Jensen media in order to determine their cultural characteristics, and molecular identification of the strains was performed first by polymerase chain reaction (PCR) assay followed by reverse hybridization (GenoType Mycobacterium CM and AS kits, Hain Lifescience, Germany). The Cohen's kappa statistical coefficient was used to evaluate the degree of agreement of the phenotypic with the molecular method.

Results: Of 62 NTM isolates tested with the molecular method, $54(87.1 \%)$ tested positive and the main species identified were Mycobacterium fortuitum (52\%), followed by Mycobacterium abscessus (13\%) alone or in combination with other species. Thirty-six $(58.1 \%)$ of the 62 NTM isolates were identified phenotypically, out of which $31(86.1 \%)$ were correctly identified by molecular method. The comparison of molecular and phenotypic methods revealed a good concordance, allowing the use of cultural patterns as identification tests in resource limited settings. However, MTBc isolates were identified among the NTM isolates, indicating that even if the rapid test for detection of MPT64 antigen is quite accurate, it could lack sensitivity and specificity in some cases.

Conclusion: Mycobacterium fortuitum and M. abscessus were identified as the main NTM species circulating in Abidjan but there is need for additional evaluation of MPT64 antigen detection assay for MTBC.
\end{abstract}

Keywords: non-tuberculous mycobacteria, identification, PCR, GenoType CM/AS, culture

Copyright 2021 AJCEM Open Access. This article is licensed and distributed under the terms of the Creative Commons Attrition 4.0 International License $<$ a rel="license" href="http://creativecommons.org/licenses/by/4.0/", which permits unrestricted use, distribution and reproduction in any medium, provided credit is given to the original author(s) and the source. Editor-in-Chief: Prof. S. S. Taiwo

\section{Mycobactéries non tuberculeuses isolées chez des patients suspects de tuberculose à Abidjan, Côte d'Ivoire}

\author{
*1,2Ouassa, T., ${ }^{1} \mathrm{~N}^{\prime}$ Guessan-Kacou, M. S., et ${ }^{2}$ Kouakou, K. A. \\ ${ }^{1}$ Centre de diagnostic et de recherche sur le sida et autres maladies infectieuses (CeDReS), \\ CHU de Treichville, Abidjan \\ ${ }^{2}$ Département de Bactériologie-Virologie, Faculté de Pharmacie, Université Félix Houphouët-Boigny, Abidjan \\ *Correspondance à: timothee.ouassa@cedres.org; timouassa@yahoo.fr; 0022521258459; 0022502500078
}

\section{Résumé:}

Contexte: Outre la tuberculose causée par les espèces du complexe Mycobacterium tuberculosis (MTBc), il existe de nombreuses autres infections mycobactériennes dues à des mycobactéries non tuberculeuses (MNT). Ceux-ci sont rarement identifiés dans de nombreuses régions à ressources limitées, notamment en Afrique en raison du manque de méthodes d'identification précises. Le but de l'étude était d'identifier les espèces de MNT impliquées dans les infections respiratoires à Abidjan, en Côte d'Ivoire.

Méthodologie: Des isolats identifiés en routine comme étant des MNT par la détection de l'antigène MPT64 entre 
2015 et 2018 au Centre de diagnostic et de recherche sur le sida et autres maladies infectieuses (CeDReS) sis au sein du CHU de Treichville, ont été inclus dans l'étude. Les souches bactériennes ont été réisolées sur trois milieux de Lowenstein-Jensen différents afin de déterminer leurs caractéristiques culturales, et l'identification moléculaire des souches a d'abord été réalisée par un test réaction de polymérisation en chaîne (PCR) suivi d'une hybridation inverse (kits GenoType Mycobacterium CM et AS, Hain Lifescience, Allemagne). Le test statistique kappa de Cohen a été utilisé pour évaluer le degré d'accord entre le phénotype et la méthode moléculaire. Résultats: Sur 62 isolats de NTM testés avec la méthode moléculaire, $54(87,1 \%)$ ont été trouvés positifs les principales espèces identifiées étant Mycobacterium fortuitum ( $52 \%)$, suivi de Mycobacterium abscessus (13\%) seul ou en association avec d'autres espèces. Trente-six $(58,1 \%)$ des 62 isolats de MNT ont été identifiés phénotypiquement, parmi lesquels $31(86,1 \%)$ ont été correctement identifiés par la méthode moléculaire. La comparaison des méthodes moléculaires et phénotypiques a révélé une bonne concordance, permettant l'utilisation de caractères culturaux comme tests d'orientation dans des zones à ressources limitées. Cependant, des isolats de MTBc ont été identifiés parmi les isolats de MNT, indiquant que même si le test rapide de détection de l'antigène MPT64 est assez précis, il pourrait manquer de sensibilité et de spécificité dans certains cas. Conclusion: Mycobacterium fortuitum et $M$. abscessus ont été identifiés comme les principales espèces de MNT circulant à Abidjan mais il est nécessaire de procéder à une évaluation supplémentaire du test de détection de l'antigène MPT64 pour l'identification des MTBc.

Mots clés: mycobactéries non tuberculeuses, identification, PCR, GenoType CM/AS, culture

\section{Introduction:}

The fight against mycobacterial infections is mainly focused on tuberculosis (TB) which is caused by species belonging to the Mycobacterium tuberculosis complex (MTBc). However, many other species called nontuberculous mycobacteria (NTM) or mycobacteria other than tuberculosis (MOTT) are generally encountered in the environment and can also be involved in human infections (mycobacterioses) (1). NTM were previously considered as occurring mostly in patients with immune deficiency, and particularly in persons living with HIV/AIDS (PLHIV) (2). However, NTM are currently known to cause various diseases, also in immunocompetent people, including ulcerative forms such as Buruli ulcer (3). NTM can be responsible for respiratory tract infections as well (1). Therefore, there is a need for accurate identification of NTM and discrimination from pulmonary tuberculosis.

In Ivory Coast, as in many parts of Africa where microscopy is the main tool for the diagnosis of TB and other mycobacterial infections, only few studies have investigated the implication of NTM in pulmonary diseases and $a$ few of them have identified species involved in those infections (4). Indeed, phenotypic identification methods using cultural and biochemical characteristics have low sensitivity, while molecular tests are more accurate but costly. Thus, the identification process is mainly limited to the differentiation between MTBC and NTM strains in these settings. As a consequence, there are usually no options provided to clinicians for the management of patients with suspected NTM infections (5). However, the determination of the major NTM species circulating in a given area can be useful as specific treatments have been identified for most of them (6). The aim of the study is to determine NTM species involved in respiratory infections in Abidjan, Ivory Coast.

\section{Materials and method:}

\section{Study setting}

The study was conducted at the mycobacteriology unit of the Centre for Diagnosis and Research on AIDS and other Infectious Diseases (CeDReS), located at the University Hospital of Treichville, Abidjan, Ivory Coast.

\section{Routine isolation of Mycobacteria strains}

From January 2015 to January 2019, mycobacterial strains isolated from patients with suspected TB and routinely collected in our laboratory were tested. These strains were isolated following processing of the patients' samples (respiratory and non-respiratory) first by decontamination/digestion process and then inoculating both Lowenstein-Jensen (LJ) (Biorad, Marne-La-Coquette, France) and BACTEC MGIT (Becton Dickinson, Sparks, MD, USA). The $L J$ tubes were incubated in aerobic conditions at $37^{\circ} \mathrm{C}$ while MGIT tubes were incubated in MGIT 960 analyzer (Becton Dickinson, Sparks, MD, USA). Bacterial growths on L] medium were monitored daily during the first week and then weekly for 6 weeks, and hourly on BACTEC MGIT 960 automated incubator.

For each positive culture, the isolate was identified by Ziehl-Neelsen (ZN) staining and detection of MPT64 antigen (SD BIOLINE TB Ag MPT64 Rapid, Standard Diagnostics, Seoul, South Korea) according to the manufacturer's instructions. This lateral flow assay is based on the detection of MPT64 antigen specific for MTBc isolates. Briefly, $100 \mu \mathrm{l}$ of the liquid culture was put unto the sample well. After 15 min, a reactive result (MTBC) was characterized by the presence of two pink bands, the test " $T$ " band, and " $C$ " zone (control band) that confirmed the test validity. The presence of only the " $C$ " (control band) indicated a non-reactive result, which in the presence of acid-fast bacilli on ZN staining, indicated the presence of NTM. M. tuberculosis 
H37Rv strain and a well-characterized external quality assessment (EQA) isolate of $M$. fortuitum obtained from the South African National Health Laboratory Service (NHLS) TB culture proficiency testing, were used as positive and negative controls respectively. All the isolates displaying non-reactive results for the determination of MPT64 antigen were identified as NTM and selected isolates out of these were included in the study.

\section{Phenotypic identification and characterization of NTM isolates}

The phenotypic characterization performed on 62 consecutively selected isolates, consisted the analysis of cultural patterns i. e. time of growth and aspects of the colonies (eugonic or dysgonic, presence or absence of pigment) following inoculation of three Lowenstein-Jensen media, with two of the culture tubes covered with aluminum foil in order to allow growth in the absence of light. The LJ tubes were incubated in aerobic conditions at $37^{\circ} \mathrm{C}$ for 6 weeks during which bacteria culture growth was monitored daily.

\section{Molecular identification of NTM isolates}

The molecular identification of the 62 NTM isolates was carried out by using GenoType Mycobacterium CM Ver 2.0 and GenoType Mycobacterium AS kits (Hain Lifescience, Manheim, Germany) according to the manufacturer's instructions. Briefly, molecular identification of the strains started by DNA extraction, followed by an amplification step with a polymerase chain reaction, and then reverse hybridization.

\section{DNA extraction:}

The GenoLyse ${ }^{R}$ kit was used for extraction by adding into $2 \mathrm{ml}$ Eppendorf tube, $5 \mu \mathrm{l}$ of internal control (IC) to $1 \mathrm{ml}$ of liquid culture. After centrifugation at 10000 rpm for 15 mins, the pellet was resuspended in $100 \mu$ l of lysis buffer and incubated at $95^{\circ} \mathrm{C}$ for 5 minutes. One hundred microliters of the neutralizing buffer were then added and the mix spined at $13000 \mathrm{rpm}$ for 5 minutes. The supernatant, containing DNA was used for the PCR.

\section{PCR assay}

For the amplification using GenoType MTBDRplus Ver 2.0 test in a thermal cycler, 10 $\mu \mathrm{l}$ of amplification mix $A$ (AM-A, containing buffer, nucleotides and Taq polymerase), $35 \mu \mathrm{l}$ of amplification mix $B$ (AM-B, containing salts, specific primers and dye) and $5 \mu$ of DNA in a final volume of $50 \mu \mathrm{l}$ was used. The amplification protocol included $15 \mathrm{~min}$ of initial denaturation at $95^{\circ} \mathrm{C}$, followed by 10 cycles, comprising 30 secs at $95^{\circ} \mathrm{C}$ and 2 mins at $58^{\circ} \mathrm{C}$ plus 20 additional cycles comprising 25 secs at $95^{\circ} \mathrm{C}, 40$ secs at $53^{\circ} \mathrm{C}$, and 40 secs at $70^{\circ} \mathrm{C}$, and a final extension at $70^{\circ} \mathrm{C}$ for 8 mins.

\section{Hybridization and detection:}

Hybridization and detection were performed in TwinCubator system (Hain Life science, Manheim, Germany), with a total of 17 probes in the GenoType Mycobacterium CM and GenoType Mycobacterium AS kits, including amplification and hybridization controls for quality check of the procedures. The first step consisted mixing $20 \mu \mathrm{l}$ of the amplicons with $20 \mu \mathrm{l}$ of denaturing reagent for $5 \mathrm{~min}$ in separate wells of a plastic reaction plate. The strips were then put in each well and $1 \mathrm{ml}$ of prewarmed hybridization buffer added, allowing the hybridization process to occur at $45^{\circ} \mathrm{C}$ for 30 mins, followed by a stringent washing step. Streptavidin conjugated with alkaline phosphatase was added for 30 mins at room temperature and washed before addition of the substrate for 5 mins. A final washing was performed and strips were air dried and sticked on a paper form.

\section{Statistical analysis}

The Cohen's kappa statistical coefficient was calculated to evaluate the degree of accuracy and reliability of the phenotypic method. The test was interpreted as follows; 0.01-0.20 slight agreement; $0.21-0.40$ fair agreement; $0.41-0.60$ moderate agreement; 0.61-0.80 substantial agreement; $0.81-1.00$ almost perfect or perfect agreement

\section{Ethical clearance}

The study was conducted on bacterial strains routinely collected in the laboratory. Therefore, no ethical clearance was needed.

\section{Results:}

During the period of study, 4750 samples were routinely analyzed for the diagnosis of mycobacterial infections and 1121 were positive on culture, including 155 NTM isolates. Sixty-two of the 155 NTM isolates were sub-cultured for phenotypic identification and characterization on the three $\mathrm{LJ}$ media, and $36(58.1 \%)$ of these were successfully sub-cultured. Of these 36 isolates, 31 (86.1\%) were correctly identified by molecular method. In 23 of the 31 (74\%) isolates, dysgonic colonies were observed, and 94\% (29/31) of the colonies were non-chromogenic while $6 \%$ (2/31) produced pigment after exposure to light (photochromogenic strains). Most of the isolates had a growth period between 1 and 7 days $(84 \%, 26 / 31)$.

Of the 62 NTM isolates tested by the molecular method, 54 (87.1\%) isolates were confirmed to be valid NTM strains. Of these, the main species identified were $M$. fortuitum (52\%, 28/54), followed by $M$. abscessus $(13 \%, 7 / 54), M$. intracellulare $(11 \%, 6 / 54)$ and $M$. avium $(6 \%, 3 / 54)$. Surprisingly, MTBC strains were identified in four cases (alone or 
in association) and species combination were observed in five cases (Fig 1). The comparison of phenotypic and molecular methods gave a kappa value of 0.94 for both pigment production (Table 1 ) and growth time (Table 2).

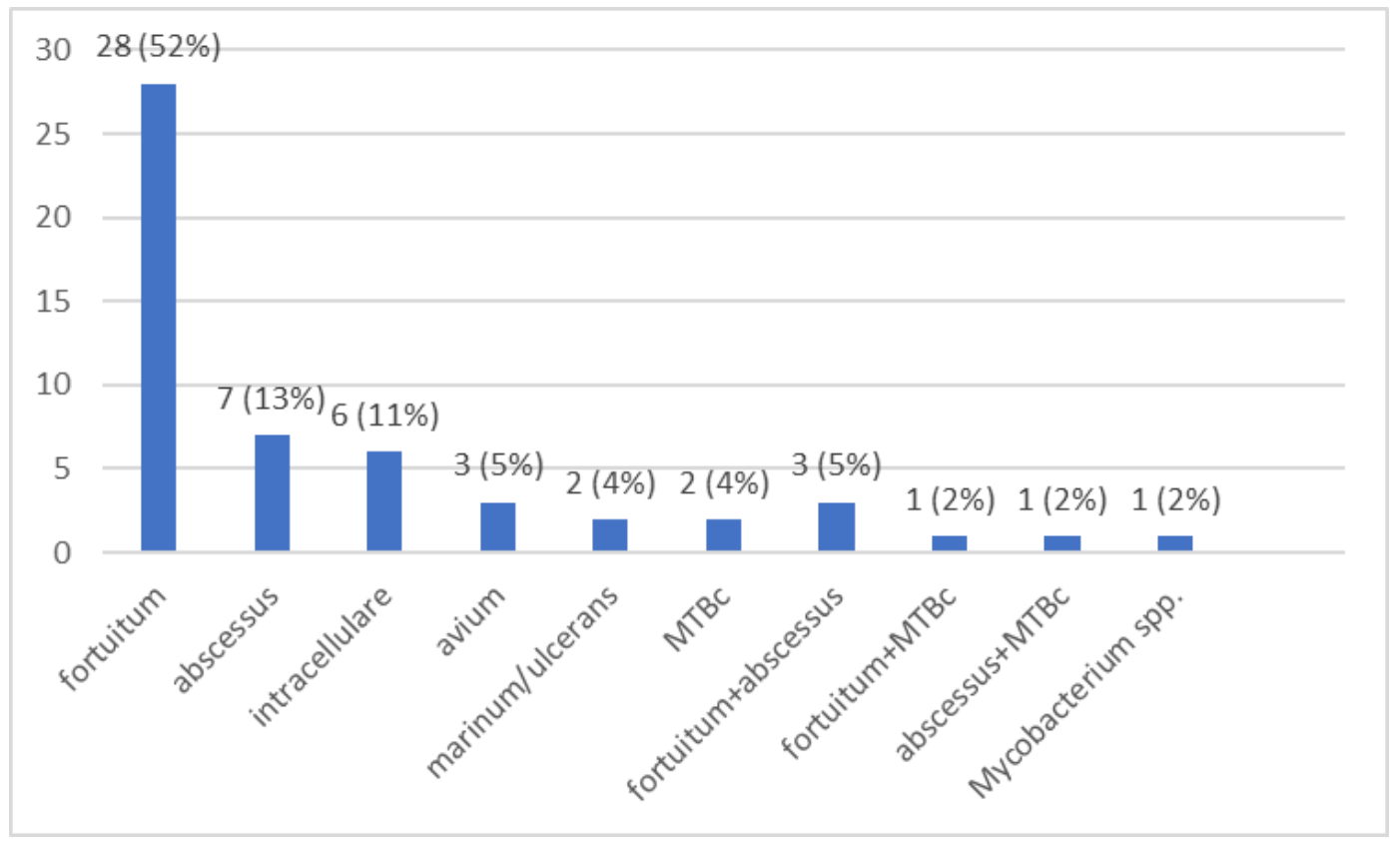

Fig 1: Non tuberculous mycobacteria species identified

Table 1: Molecular identification and pigments production

\begin{tabular}{|c|c|c|c|c|c|c|c|c|c|}
\hline Mycobacteria & fort & absc & intra & avium & $\begin{array}{c}\text { marin/ } \\
\text { ulcer }\end{array}$ & $\begin{array}{c}T b \\
c p / x\end{array}$ & $\begin{array}{c}\text { absc/ } \\
\text { Tb cplx }\end{array}$ & $\begin{array}{c}\text { absc/ } \\
\text { fort }\end{array}$ & Total \\
\hline Non chromogenic & 19 & 2 & 3 & 0 & 1 & 0 & 1 & 3 & 29 \\
\hline Photochromogenic & 1 & 0 & 1 & 0 & 0 & 0 & 0 & 0 & 2 \\
\hline Scotochromogenic & 0 & 0 & 0 & 0 & 0 & 0 & 0 & 0 & 0 \\
\hline Total & 20 & 2 & 4 & $\mathbf{0}$ & 1 & $\mathbf{0}$ & 1 & 3 & 31 \\
\hline
\end{tabular}


Table 2: Molecular identification and growth delay

\begin{tabular}{cccccccccc}
\hline Days & Fort & absc & Intra & Avium & $\begin{array}{c}\text { marin/ } \\
\text { ulcer }\end{array}$ & Tb cplx & $\begin{array}{c}\text { absc/ } \\
\text { Tb cplx }\end{array}$ & $\begin{array}{c}\text { absc/ } \\
\text { fort }\end{array}$ & \begin{tabular}{c} 
Total \\
\hline $1-7$
\end{tabular} \\
\hline & 19 & 2 & 1 & 0 & 0 & 0 & 1 & 3 & 26 \\
$8-14$ & 0 & 0 & 0 & 0 & 0 & 0 & 0 & 0 & 0 \\
$15-21$ & 1 & 0 & 2 & 0 & 0 & 0 & 0 & 0 & 3 \\
$22-28$ & 0 & 0 & 1 & 0 & 1 & 0 & 0 & 0 & 2 \\
$>29$ & 0 & 0 & 0 & 0 & 0 & 0 & 0 & 0 & 0 \\
Total & $\mathbf{2 0}$ & $\mathbf{2}$ & $\mathbf{4}$ & $\mathbf{0}$ & $\mathbf{1}$ & $\mathbf{0}$ & $\mathbf{1}$ & $\mathbf{3}$ & $\mathbf{3 1}$ \\
\hline fort = fortuitum; absc = abscessus; intra = intracellulare; marin/ulcer = marinum/ulcerans; Tb cplx = tuberculosis complex; Grayed cell = discordance
\end{tabular}

\section{Discussion:}

This study provides a more recent insight about the distribution of NTM in Ivory Coast as the previous study was conducted more than 15 years ago (7). It also revealed that most of the isolates were fast growing mycobacteria ( $M$. fortuitum and $M$. abscessus), slow growing MTN being less frequent (M. intracellulare and $M$. avium). These results are quite similar to those observed in a metanalysis related to NTM isolates from respiratory samples in sub-Saharan African countries. Indeed, all these pathogens belonged to the top four species identified in various studies conducted in this area, with $M$. avium complex (MAC) being more frequently isolated (4).

Among the species belonging to this complex, $M$. intracellulare and $M$. avium are those frequently involved in opportunistic mycobacterial infections in PLHIV (2). It is noteworthy that although these mycobacteria were previously identified by the routine laboratory cultures as NTM, some isolates belonging to MTBC were also identified among them. This indicates that even if lateral flow antigen detection tests are quite accurate, they could lack sensitivity and specificity in some cases as reported by different authors (8). A relatively good concordance between the molecular test and phenotypic cultural patterns was also observed in our study, which implies that phenotypic culture method could be used as identification tests in low resource settings.

\section{Acknowledgements:}

The authors acknowledge the laboratory technicians (Yeo S, Keita A, and Konin A) who contributed to the isolation of the strains.

\section{Authors contributions:}

TO designed the study, performed the tests and drafted the manuscript, MSNK and KAK performed the tests and revised the manuscript. All authors read and approved the final manuscript.

\section{Previous presentation:}

The findings of this study were presented in part at the first congress of the Societé Ivoirienne de Microbiologie (Ivorian Society for Microbiology), in Yamoussoukro, Ivory Coast on November, 29th 2019.

\section{Conflicts of interest:}

Authors declare no conflict of interest.

\section{References:}

1. Marras, T. K., and Daley, C. L. Epidemiology of human pulmonary infection with non-tuberculous mycobacteria. Clin Chest Med. 2002; 23 (3): 553-567.

2. Karakousis, P. C., Moore, R. D., and Chaisson, R. E. Mycobacterium avium complex in patients with HIV infection in the era of highly active antiretroviral therapy. Lancet Infect Dis. 2004; 4 (9): 557 565.

3. van der Werf, T. S., van der Graaf, W. T., Tappero, J. W., and Asiedu, K. Mycobacterium ulcerans infection. Lancet Lond Engl. 1999; 354 (9183): 1013-1018.

4. Okoi, C., Anderson, S. T. B., Antonio, M., Mulwa, S. N., Gehre, F., and Adetifa, I. M. O. Nontuberculous Mycobacteria isolated from pulmonary samples in sub-Saharan Africa-a systematic review and meta-analyses. Sci Rep. 2017; 7 (1): 12002.

5. Maiga, M., Siddiqui, S., Diallo, S., et al. Failure to recognize non tuberculous mycobacteria leads to misdiagnosis of chronic pulmonary tuberculosis. PLoS One. 2012; 7 (5): e36902. 
6. Ryu, Y. J., Koh, W. J., and Daley, C. L. Diagnosis and Treatment of Nontuberculous Mycobacterial Lung Disease: Clinicians' Perspectives. Tuberc Respir Dis. 2016; 79 (2): 74-84.

7. Bonard, D., Messou, E., Seyler, C., Vincent, V., Gabillard, D., and Anglaret, X. High incidence of atypical mycobacteriosis in African HIV-infected adults with low CD4 cell counts: a 6-year cohort study in Côte d'Ivoire. AIDS Lond Engl. 2004; 18 (14): 1961-1964.

8. Yin, X., Zheng, L., Lin, L., et al. Commercial MPT64-based tests for rapid identification of Mycobacterium tuberculosis complex: a metaanalysis. J Infect. 2013; 67 (5): 369-377. 